\title{
Facile Preparation and Properties Study of CL-20/TATB/VitonA Composite Microspheres by a Spray-Drying Process
}

\author{
Conghua Hou $\mathbb{D}^{1},{ }^{1}$ Congcong Li $\mathbb{D}^{1},{ }^{1}$ Xinlei Jia, ${ }^{2}$ Yuanping Zhang, ${ }^{1}$ and Shimin Zhang $^{1}$ \\ ${ }^{1}$ School of Environment and Safety Engineering, North University of China, Taiyuan 030051, China \\ ${ }^{2}$ Department of Chemical Engineering and Safety, Binzhou University, Binzhou, Shandong 256600, China \\ Correspondence should be addressed to Congcong Li; lcc18834161634@163.com
}

Received 15 October 2019; Revised 10 March 2020; Accepted 18 March 2020; Published 7 April 2020

Academic Editor: Laura Martinez Maestro

Copyright (C) 2020 Conghua Hou et al. This is an open access article distributed under the Creative Commons Attribution License, which permits unrestricted use, distribution, and reproduction in any medium, provided the original work is properly cited.

\begin{abstract}
To improve the safety of ammonium nitrate explosives, a one-step method involving the formation of CL-20/TATB/VitonA composite microspheres using spray drying is presented. The crystal morphology, particle size, crystal structure, thermal properties, and sensitivity of raw materials of CL-20 and TATB, CL-20/VitonA particles, and CL-20/TATB/VitonA microspheres were characterized by scanning electron microscopy (SEM), X-ray diffraction (XRD), differential scanning calorimetry (DSC), and impact and friction sensitivity tests. The SEM results show that the prepared CL-20/TATB/VitonA composites with micro-/nanoscale grain size have a good spherical shape. Thermal analysis data show that TATB coating enhances the thermal stability of CL-20/VitonA. The impact and friction sensitivity results show that superior mechanical properties of these composite microspheres could be maintained. Obviously, this approach is an effective desensitization technique to prepare composite microspheres for explosives.
\end{abstract}

\section{Introduction}

Currently, as technology and weapon systems continue to evolve, energetic materials should not only have high power, high precision, and long range for weapon firepower systems but also maintain relatively high safety in other environments [1]. However, existing explosives including 2,4,6,8,10,12hexanitro-2,4,6,8,10,12-hexaazaiso-wurtzitane(CL-20), 1,3,5,7teranitro-1,3,5,7-tetrazocine (HMX), and hexahydro-1,3,5trinitro-1,3,5-triazine (RDX) exhibiting high energy density, good heat resistance, stable detonation, and high detonation speed usually suffer from high mechanical sensitivity and poor shock wave sensitivity [2]. During the past decades, many studies have been devoted to the desensitization of nitramine explosives, usually using refinement, coating, and eutectic techniques to reduce sensitivity [3-7]. At present, coating is the most convenient way to improve the comprehensive performance of explosives, and common coating methods include water suspension method, crystallization coating method, spray-drying method, and in situ polymerization [8-10]. Our research is based on spray-drying methods described above.
CL-20 is a nonaromatic cyclic nitramine with high density, high energy, high detonation velocity, and high detonation pressure, and it is the most attractive high-energydensity compound (HEDC) with promising application in the fields of agents, formulated explosives, and propellants $[11,12]$. Unfortunately, the high mechanical sensitivity and poor shock wave sensitivity significantly limit its further application. Hence, it is essential to increase the insensitivity of CL-20 to satisfy the high energy and high safety requirements of modern weapons for ammunition. In contrast, TATB (1,3,5-triamino-2,4,6-trinitrobenzene) is an excellent insensitive explosive owing to its high melting point, high crystal density, low mechanical sensitivity, and good heat resistance. Therefore, it is widely used in atomic bombs, nuclear warheads, high-polymer-bonded explosives, etc. [13].

To improve the safety of CL-20 while preserving its high energy yield, Li et al. [14] prepared CL-20/TATB nanocomposites using the fast antisolvent crystallization method. The results indicated that CL-20 in the nanocomposite took the form of an $\alpha$ polymorph, and the nanocomposite had much lower sensitivity. Xu et al. [15] prepared nano-TATB 
particles by ultrasonic-assisted spray method and used molecular dynamics simulation to select the best binder VitonA. The composite particles had higher thermal stability and lower mechanical sensitivity. Xu et al. [16] prepared a CL-20/TATB cocrystal explosive through a solvent/nonsolvent rapid nucleation process. The results indicated that the cocrystal was a new substance rather than a mixture of raw materials, and it had excellent thermal stability, high energy release, and lower mechanical sensitivity.

Spray-drying technology has been used in the industry for more than 100 years. At first, due to the low thermal efficiency of this process, it was limited to a small number of products such as milk powder and egg powder; as technology and equipment continue to evolve, the application range of spray-drying technology has been continuously expanded. It has been widely used in the chemical industry, food industry, pharmaceuticals and biochemical industry, material processing, and other fields. In recent years, spray-drying technology has been applied to the field of energetic materials. Ji et al. [17] prepared $\mathrm{HMX} / \mathrm{F}_{2602}$ using a suspension spray-drying method. The test results showed that $\mathrm{F}_{2602}$ was successfully coated on the surface of HMX crystals. Compared with the raw material HMX, the impact and friction sensitivities were reduced. An et al. [18] prepared the NC/HMX composite using the codissolved spray-drying method. The impact sensitivity was significantly reduced, and the decomposition peak temperature appeared earlier than that of the raw HMX. Wang et al. [19] coated VitonA and GO on the surface of HMX crystals using the spraydrying method. Compared with HMX/VitonA, the composite particles showed better thermal stability, and the impact and shock wave sensitivities of the composite decreased. In previous reports, the most conventional method for preparing CL-20/TATB composite materials is usually the water suspension method. VitonA as a fluorine rubber has significant advantages such as chemical stability, good compatibility with explosives, and low surface energy [20]. However, as CL-20 and VitonA are often codissolved in common solvents, CL-20/TATB/VitonA composites can be difficult to prepare using the water suspension method. Therefore, it is a better choice to prepare CL-20/TATB/VitonA using spray drying.

In this study, we prepared CL-20/TATB/VitonA composite microspheres by suspension spray drying for the first time. The preparation method is simple, efficient, and scalable. The formation mechanism, crystal morphology, structure, thermal properties, and sensitivity characteristics of composite microspheres were systematically investigated in detail.

\section{Experiment}

2.1. Materials. Raw CL-20 and TATB were provided by Gansu Yinguang Chemical Industry Group Co., Ltd. A copolymer of vinylidene fluoride and hexafluoropropylene (VitonA) was produced by Sichuan Chenguang Chemical Company. Ethyl acetate was provided by Nanjing Chemical Reagent Co., Ltd. Pure water was provided by the pure water supply of Taiyuan Iron and Steel Co., Ltd.
2.2. Sample Preparation. CL-20/TATB/VitonA composite microspheres were prepared by suspension spray drying. The experimental device is shown in Figure 1. First, $2 \mathrm{~g}$ of CL-20, different content of TATB, and $0.1 \mathrm{~g}$ of VitonA were dissolved in ethyl acetate, and the mixture was stirred well to form an explosive suspension solution. The inlet air temperature and outlet air temperature were set to $85^{\circ} \mathrm{C}$ and $50^{\circ} \mathrm{C}$, respectively, during the preparation. The liquid inlet rate was controlled at $300 \mathrm{~mL} / \mathrm{h}$, and the cyclone wind speed and spray drying gas flow rate $\left(\mathrm{N}_{2}\right)$ were $35 \mathrm{~m}^{3} / \mathrm{h}$ and $360 \mathrm{~L} / \mathrm{h}$, respectively. Finally, the spray drying gas $\left(\mathrm{N}_{2}\right)$ and the composites were separated using a cyclone, and CL-20/TATB/VitonA composite microspheres were collected in a glass collector. Under the same experimental conditions, the composite microspheres with $10 \%, 20 \%$, and $30 \%$ TATB contents were named as sample-1, sample-2, and sample-3, respectively.

2.3. Characterization. The morphology and size distribution of raw materials and composite microspheres were examined using a MIRA3 LMH scanning electron microscope (TESCAN, Czech Republic). The as-obtained samples were dispersed on conductive carbon adhesive tape to attach to a SEM stub and then gold coated for SEM investigation. Powder X-ray diffraction was used to visualize the changes in the crystal structure of the samples. PXRD patterns were recorded using a DX-2700 (Dandong Haoyuan Corporation, Liaoning, China) X-ray diffractometer at a voltage of $40 \mathrm{kV}$ and a current of $30 \mathrm{~mA}$ using $\mathrm{Cu} K \alpha$ radiation at $\lambda=1.5418 \AA$.

Thermal analysis was performed on a differential scanning calorimeter (DSC-131, France SETARAM Corporation, Shanghai, China) at a heating rate of $10^{\circ} \mathrm{C} / \mathrm{min}$. The impact sensitivity was determined with an ERL Type 12 drop hammer. The testing conditions are as follows: drop weight of $2.500 \pm 0.002 \mathrm{~kg}$, sample mass of $35 \pm 1 \mathrm{mg}$, ambient temperature of $20^{\circ} \mathrm{C}$, and relative humidity of $30 \%$. The test was carried out 25 times to characterize the impact sensitivity of the samples. The friction sensitivity of the samples was tested with a WM friction instrument. In each determination, 25 samples were tested, and an explosion probability $(P, \%)$ was obtained. The mass of sample is $20 \pm 1 \mathrm{mg}$, with a relative pressure of $4.9 \mathrm{MPa}$, swaying angle of $90^{\circ}$, and pendulum weight of $1.50 \pm 0.01 \mathrm{~kg}$.

\section{Results and Discussion}

3.1. Morphology and Size Characterization. The prepared composite microspheres have a smooth spherical structure, which is obviously different from the raw materials. The raw CL-20 shown in Figure 2(a) has mostly irregular spindle shapes, and the raw TATB shown in Figure 2(b) has irregular bulk crystals with angular surfaces. The size distribution is relatively uneven. The size distribution of raw CL-20 is $2-50 \mu \mathrm{m}$ with an average particle size of about $40 \mu \mathrm{m}$. The size distribution of raw TATB is $4-30 \mu \mathrm{m}$, with an average particle size of about $20 \mu \mathrm{m}$. Compared with composites with different TATB contents (Figures 2(d)-2(f)), the particle size of CL-20/VitonA composites shown in Figure 2(c) 


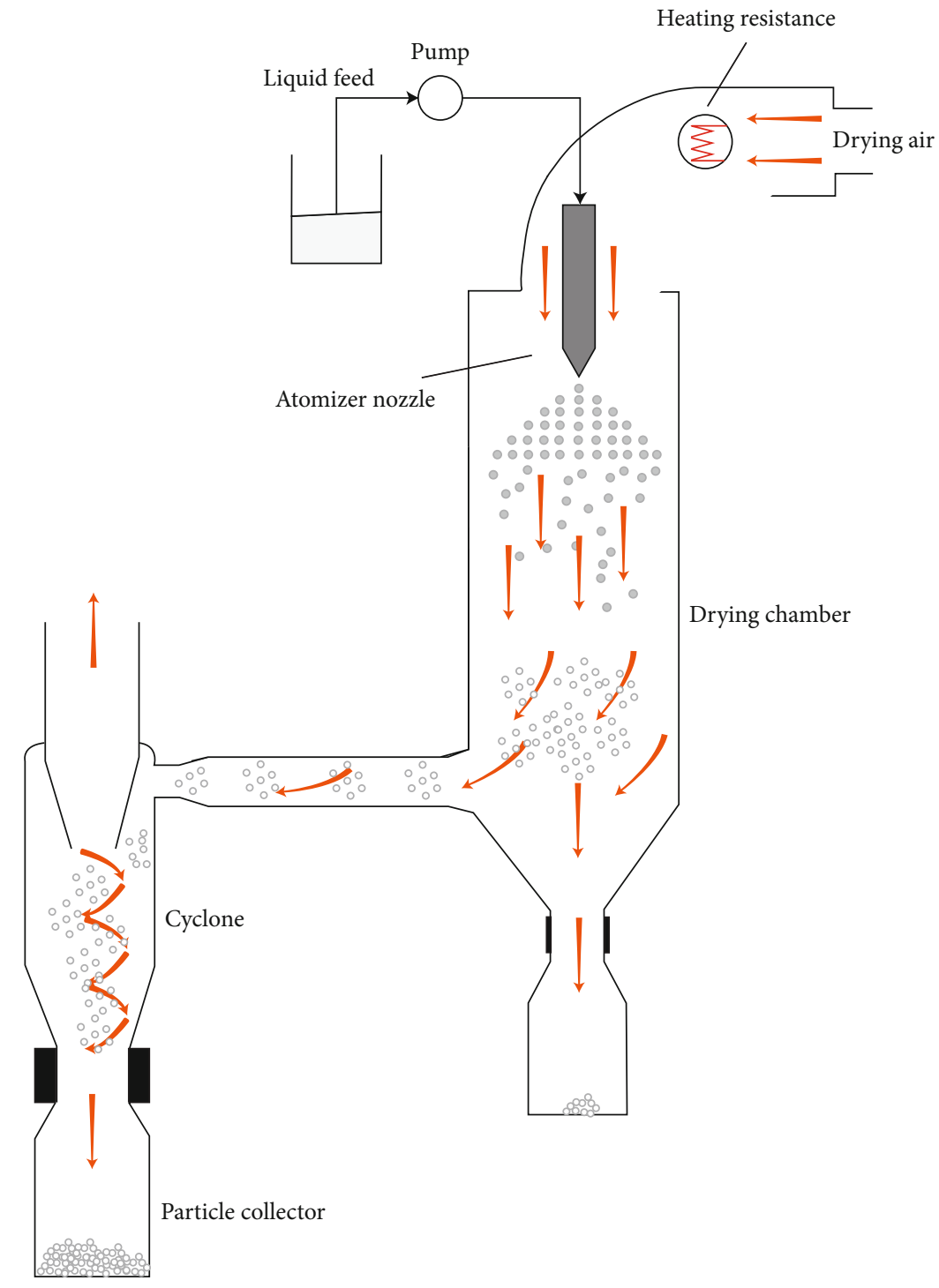

FIGURE 1: Schematic diagram of spray-drying device.

is smaller, more uniform, more regular in shape, and tend to be spherical or spheroidal. This is because no TATB component was added; CL-20 and VitonA can be codissolved in ethyl acetate. The solution is directly ejected from the nozzle, and the surface tension of droplets tends to minimize the surface as much as possible. Therefore, CL-20/VitonA is spherical in shape with a smooth surface and good spherical effect. In Figure 2(d), 10\% TATB was added, and the particle size was relatively uniform. As the surface was coated with TATB, the composite microspheres were more compact and plump, and spherical-like particles appeared. This is probably because some of the droplets could not absorb TATB uniformly, resulting in the accumulation or absence of TATB on the surface and forming a part of spherical-like particles samples. However, most of the composite microspheres still showed spherical particles with a smooth surface and perfect coating effect, and the particle size of the sample remained in the range from $500 \mathrm{~nm}$ to $2 \mu \mathrm{m}$. With the increase of TATB content, larger composite microspheres appeared, as shown in Figure 2(e). The particle size distribution of the samples became uneven, and the average particle size increased, accompanied by irregular refinement of TATB particles. This is probably because the droplets from the nozzle are atomized during spray drying. Some of the droplets are dispersed into the drying chamber, and after a series of assembly and drying, most of them are single particles with a smaller particle size, while some of them are combined with multiple droplets, forming spherical particles with a larger particle size after coating TATB. A schematic illustration of the formation process of composite microspheres is shown in Figure 3.

As shown in Figure 3, during the preliminary preparation of spray drying, first, CL-20 and VitonA were dissolved in ethyl acetate, and the mixture was ultrasonically stirred for 15-20 min, so that CL-20 and VitonA were fully dissolved and dispersed uniformly. Then, TATB was added to the mixture, and the mixture was ultrasonically stirred for more than $30 \mathrm{~min}$. Thus, TATB was continuously polished and dispersed to form a uniform suspension, which was atomized 


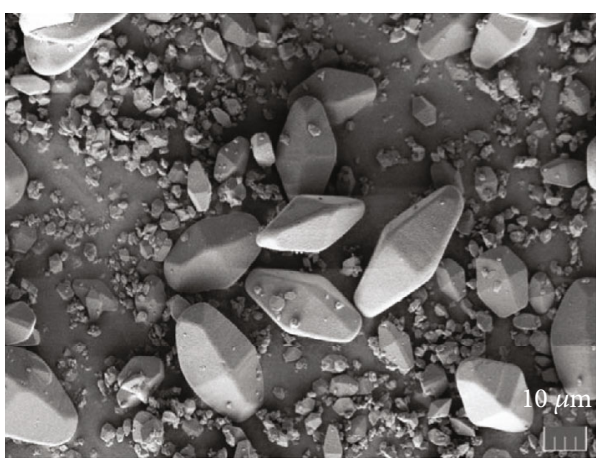

(a)

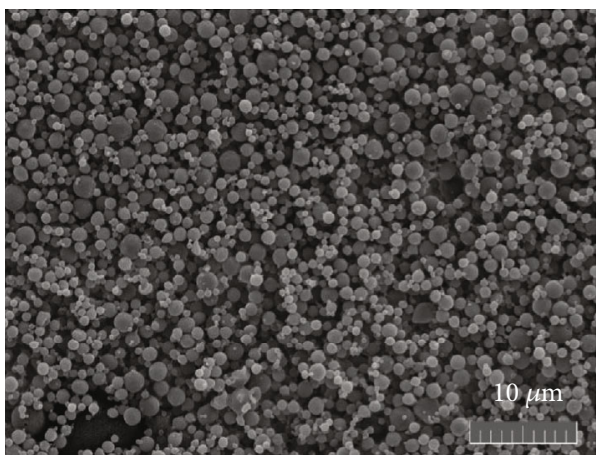

(c)

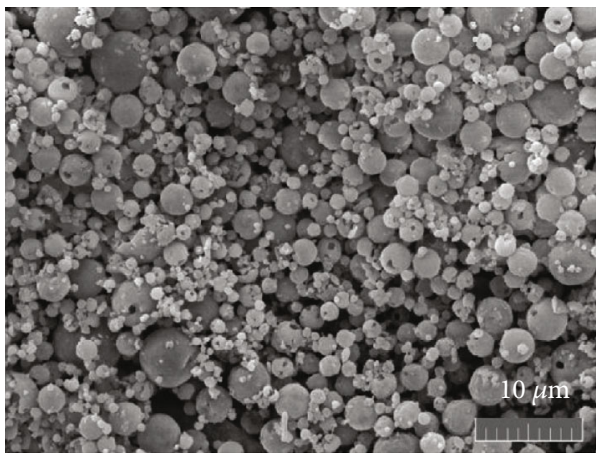

(e)

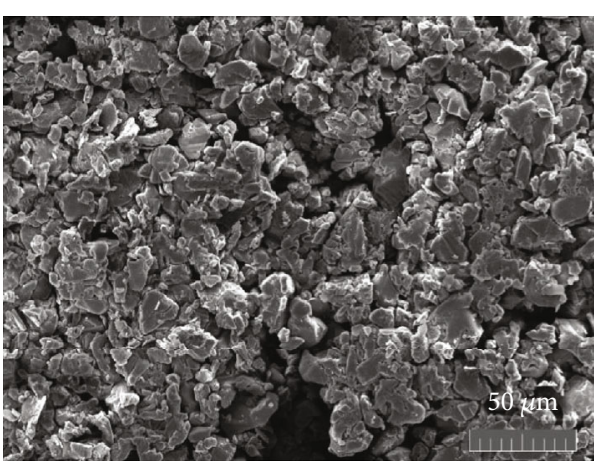

(b)

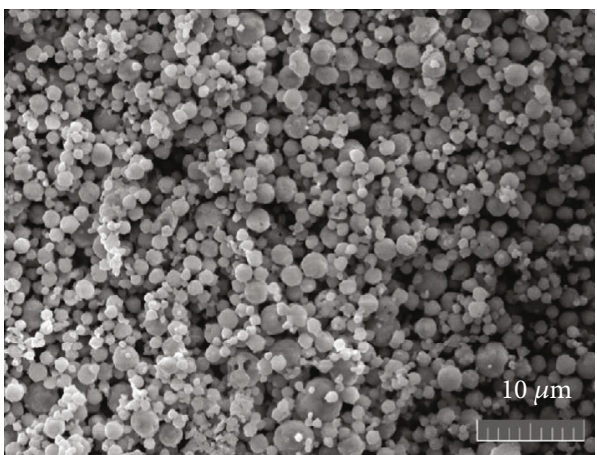

(d)

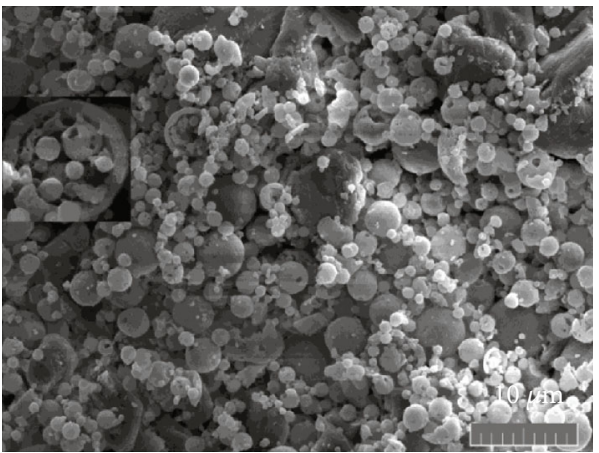

(f)

Figure 2: SEM image of raw CL-20 (a), raw TATB (b), CL-20/VitonA(c), sample-1 (d), sample-2 (e), and sample-3 (f).

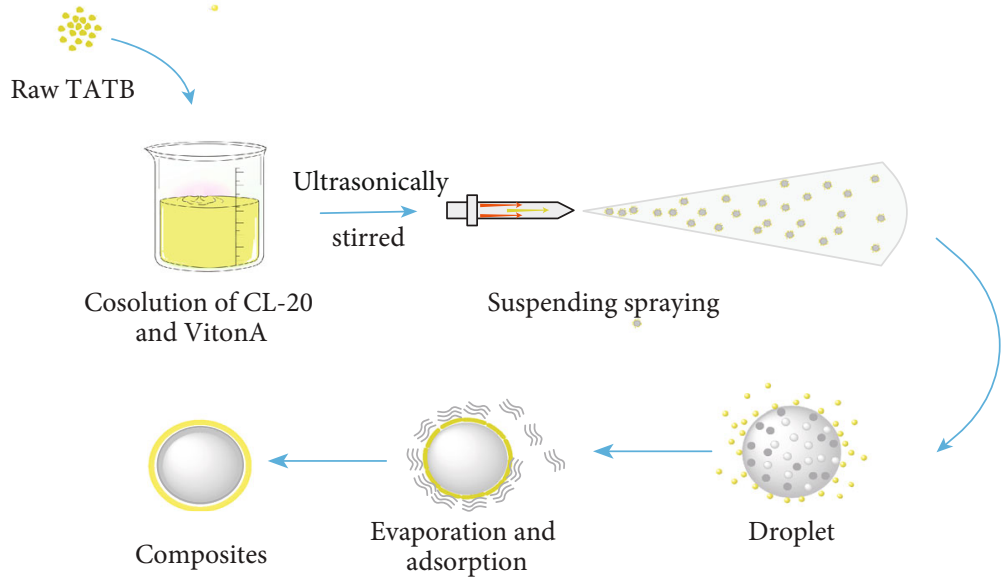

FIgURE 3: Schematic illustration of the formation of composite particles. 


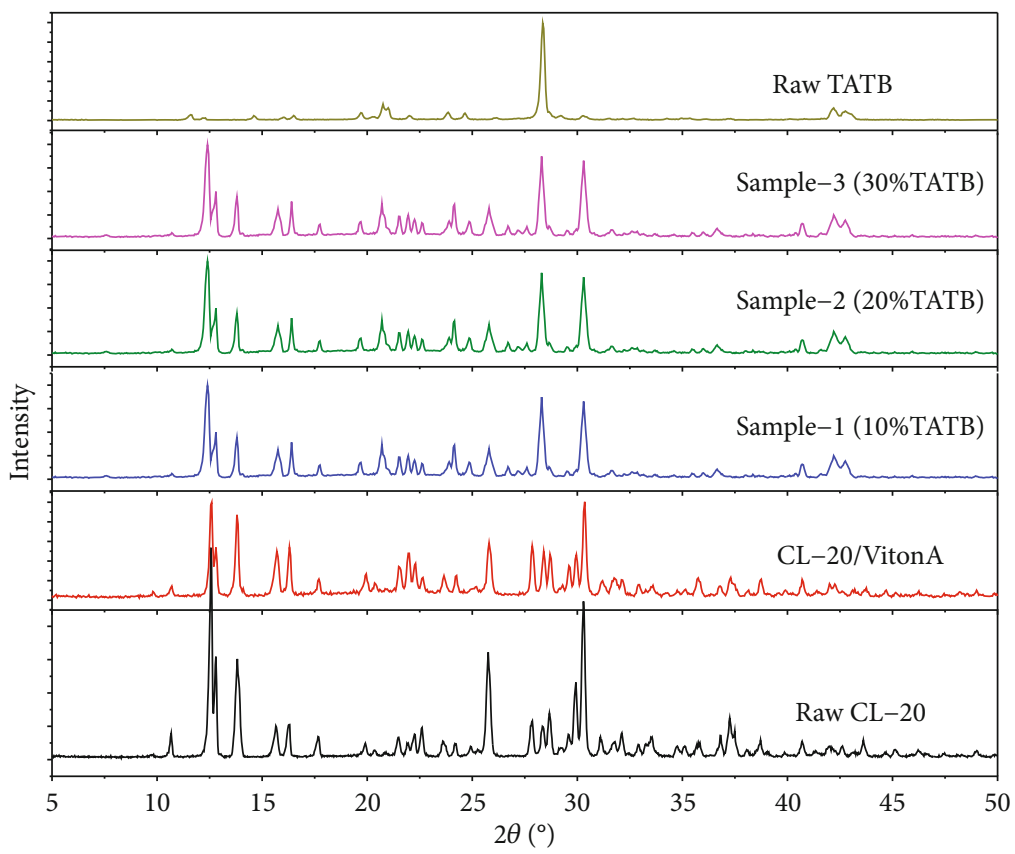

Figure 4: XRD spectra of raw CL-20, raw TATB, CL-20/VitonA, and CL-20/TATB/VitonA samples.

through a nozzle using a peristaltic pump. The spray-drying equipment in this study was a pneumatic dual-fluid nozzle with a diameter of $1 \mu \mathrm{m}$. When the suspension was atomized through the nozzle, because TATB was difficult to dissolve in ethyl acetate, it was in a uniform suspension state. The highspeed flowing nitrogen impacted the suspension under the restriction of the inner wall of the nozzle, and the TATB in the suspension was crushed and dispersed into small particles. Atomized liquid droplets were introduced into the drying chamber. The inside of the droplets contained CL-20 and VitonA molecules, which are codissolved in ethyl acetate. Owing to the adhesion of VitonA, the ultrafine TATB particles adhered to the surface of the droplets and entered the drying chamber together. When entering the drying chamber and coming into contact with heated nitrogen, the solvent evaporates quickly. During this process, the removal of the solvent caused a strong shrinkage of the droplet surface and further adhesion and aggregation of TATB [21]. In the actual spray-drying process, evaporation and formation of composite materials are completed in a very short time. Then, the composite microspheres were separated into a collection bottle through the cyclone separator.

3.2. Crystal Structure of Samples. The XRD patterns of the raw CL-20 and TATB, CL-20/VitonA, and CL-20/TATB/VitonA (samples 1-3) composite microspheres are shown in Figure 4. The main characteristic peaks of the raw CL-20 appeared at $12.57^{\circ}, 13.83^{\circ}, 25.80^{\circ}$, and $30.31^{\circ}$, corresponding to $\left(\begin{array}{ll}1 & 1-1\end{array}\right),\left(\begin{array}{lll}2 & 0 & 0\end{array}\right),\left(\begin{array}{ll}0 & 2\end{array}\right)$, and $\left(\begin{array}{ll}2 & 0-3\end{array}\right)$ crystal planes of $\varepsilon$-CL-20, respectively (PDF card 00-050-2045). The diffraction pattern of the CL-20/VitonA sample shows that the diffraction peaks correspond well with those of raw CL-20, probably because the coating mainly depends on the phase transition (liquid-solid state) of the binder and intermolecular

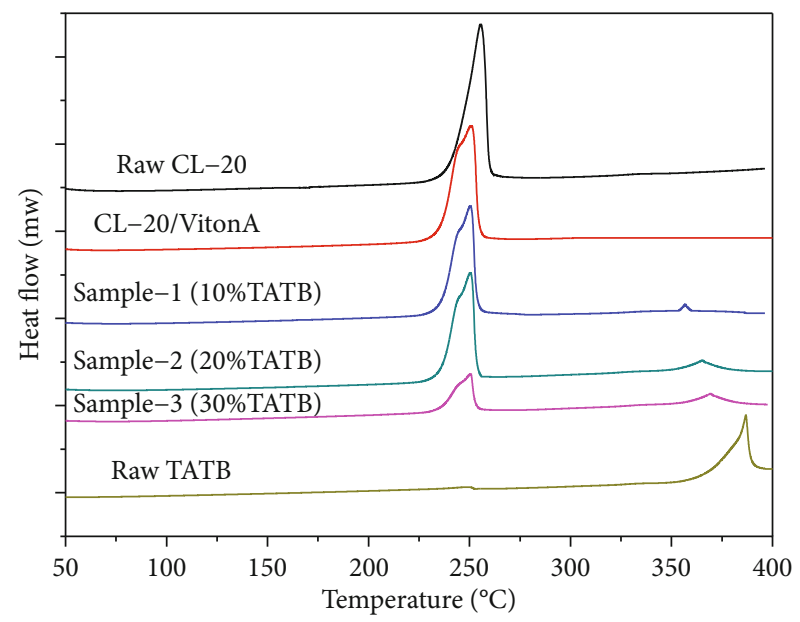

Figure 5: DSC curves of samples collected at a heating rate of $10^{\circ} \mathrm{C} / \mathrm{min}$.

TABLE 1: Thermal analysis data of samples.

\begin{tabular}{lccc}
\hline Sample & TATB (wt \%) & $\begin{array}{c}\text { Exothermic } \\
\left({ }^{\circ} \mathrm{C}\right)\end{array}$ & $\begin{array}{c}\text { Exothermic } \\
\left({ }^{\circ} \mathrm{C}\right)\end{array}$ \\
\hline Raw CL-20 & 0 & 255.59 & - \\
Raw TATB & 100 & - & 386 \\
CL-20/VitonA & 0 & 250.40 & - \\
Sample-1 & 10 & 250.49 & 359 \\
Sample-2 & 20 & 251.08 & 365 \\
Sample-3 & 30 & 251.35 & 369 \\
\hline
\end{tabular}




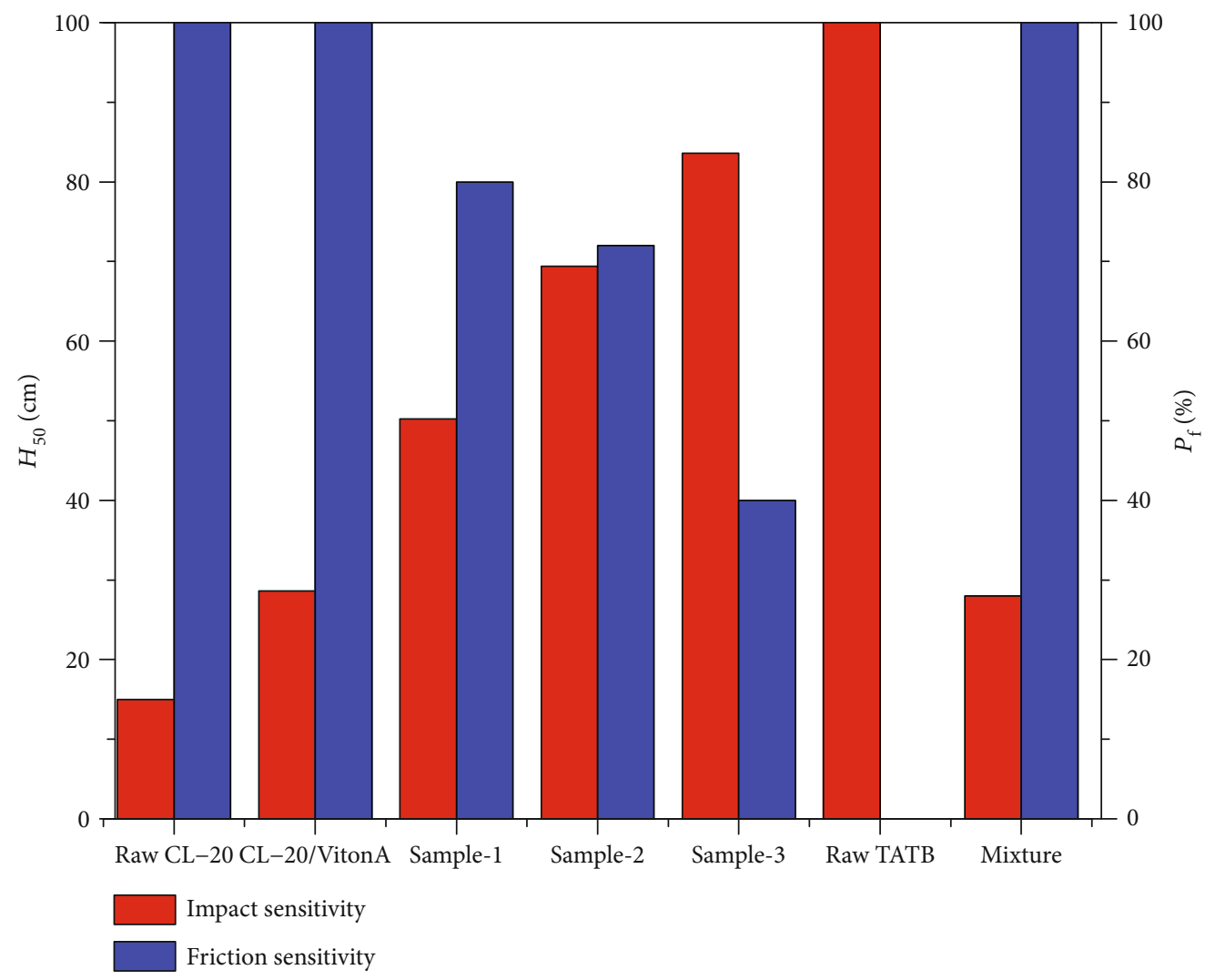

FIGURE 6: Impact and friction sensitivities of samples.

van der Waals force and hydrogen bonding to form a continuous uniform polymer binder coating on the surface of the explosive crystal without affecting the crystal form of the explosive [22]. From the diffraction curves of CL-20/TATB/VitonA composite microspheres prepared by adding different TATB contents, it can be observed that the characteristic diffraction peaks of the composite particles correspond to the raw CL-20 one to one, and typical TATB diffraction peaks can be observed on the diffraction curves. Moreover, the diffraction pattern of the composite microspheres did not change with the content of TATB. The formation of TATB coating on the surface led to the aggravation of X-ray dispersion to a certain extent, so that the characteristic diffraction peak of the sample became wider and its intensity decreased.

3.3. Thermal Performance. Thermal analysis of DSC reveals some interesting phenomena, as shown in Figure 5 and Table 1. Raw CL-20 has a strong exothermic peak at $255.59^{\circ} \mathrm{C}$, while the thermal curve of CL-20/VitonA shows an exothermic peak at $250.40^{\circ} \mathrm{C}$, which is slightly lower than the raw materials. This can be attributed to the formation of a VitonA coating on the surface of CL-20, which plays a significant role in facilitating the condensed-phase reactions and reducing the contribution of competing gas-phase processes during the thermolysis of composite particles [23]. Therefore, the decomposition rate is accelerated, resulting in an accelerated decomposition rate. On the other hand, the free radicals generated from the VitonA cleavage affected the self-heating degree of CL-20 and caused the CL-20 molecular skeleton to crack in advance. The endothermic peak temperature of samples 1-3 slightly increased than that of CL-20/VitonA, which was attributed to the formation of TATB coating to capture the free radicals generated from the VitonA cleavage [24]. Raw TATB has an endothermic peak at $386^{\circ} \mathrm{C}$, and the TATB endothermic peak of the composite particles for samples 1-3 varies with the TATB content. The higher the content of TATB, the higher the corresponding endothermic peak. However, it still appears in advance of raw TATB. The main reason is that with the increase in TATB content, more heat is needed to decompose TATB. Therefore, it is necessary to pyrolyze TATB coating at a higher temperature.

3.4. Sensitivity Study. To evaluate the safety performance of the samples, impact and friction sensitivity tests were performed, and the results are shown in Figure 6. Compared with raw CL-20, the characteristic height $H_{50}$ of CL-20/VitonA composites increased from $15 \mathrm{~cm}$ to $28.6 \mathrm{~cm}$, attributing to the compact and uniform VitonA coating on the surface of CL-20. When it underwent an external impact action, the binder would be first attacked as a buffer system to dissipate the impact energy. Raw TATB is composed of anisotropic molecular crystals with a graphite-like layered crystal structure. Compared with the same content of the CL-20/TATB mixture, the impact sensitivity for CL-20/TATB sample-1 is dramatically reduced. The $H_{50}$ value increased to $50.2 \mathrm{~cm}$. As the coating content of TATB increased, the characteristic height $H_{50}$ of the composite sample increased to 
$69.6 \mathrm{~cm}$ and $83.6 \mathrm{~cm}$, and the friction sensitivity significantly decreased with increasing content of TATB, thus significantly improving the safety performance. This is because TATB is successfully coated on the surface of CL-20, which can produce a certain buffer effect under external mechanical stimulus, thus effectively slowing down the formation of hot spots [25]. For CL-20/TATB/VitonA composite microspheres, the uniform, small particle size distribution increases the gap between themselves, which reduces the stress concentration between the particles and effectively prevents the formation of hot spots.

\section{Conclusion}

In conclusion, we propose a spray-drying technique to produce CL-20/TATB/VitonA composite microspheres with micro-/nanoscale grain size, good spherical shape, and reduced mechanical sensitivity. The formation mechanism of CL-20/TATB/VitonA microspheres is proposed. The composites showed regular-shaped composite microspheres with a diameter of $500 \mathrm{~nm}$ to $2 \mu \mathrm{m}$ and exhibited excellent properties. Thermal analysis data show that TATB coating enhances the thermal stability of CL-20/VitonA, and a higher content of TATB can help eliminate the influence of free radicals generated from the VitonA cleavage on the self-heating degree of CL-20. With the increase in TATB content, the impact and friction sensitivities decreased significantly. This study provides a certain reference for the application of high-energy and low-sensitivity ammunition in weapon firepower and rocket systems.

\section{Data Availability}

The data used to support the findings of this study are available from the corresponding author upon request.

\section{Conflicts of Interest}

The authors declare that there is no conflict of interest regarding the publication of this paper.

\section{Acknowledgments}

This research work was financially supported by the Graduate Education Innovation Project in Shanxi Province.

\section{References}

[1] V. Thottempudi, F. Forohor, D. A. Parrish, and J. M. Shreeve, "Tris (triazolo) benzene and its derivatives: high-density energetic materials," Angewandte Chemie, vol. 51, no. 39, pp. 98819885, 2012.

[2] Z. Yang, L. Ding, P. Wu, Y. Liu, F. Nie, and F. Huang, "Fabrication of RDX, HMX and CL-20 based microcapsules via in situ polymerization of melamine-formaldehyde resins with reduced sensitivity," Chemical Engineering Journal, vol. 268, pp. 60-66, 2015.

[3] W. Ji, X. Li, and J. Wang, "Preparation and characterization of CL-20/EPDM by a crystal refinement and spray drying method," Central European Journal of Energetic Materials, vol. 12, no. 4, pp. 831-840, 2015.

[4] B. Ye, C. An, Y. Zhang, C. Song, X. Geng, and J. Wang, "Onestep ball milling preparation of nanoscale CL-20/graphene oxide for significantly reduced particle size and sensitivity," Nanoscale Research Letters, vol. 13, no. 1, pp. 42-50, 2018.

[5] Z. Yang, H. Li, H. Huang, X. Zhou, J. Li, and F. Nie, "Preparation and performance of a HNIW/TNT cocrystal explosive," Propellants, Explosives, Pyrotechnics, vol. 38, no. 4, pp. 495501, 2013.

[6] H. Li, C. An, W. Guo, X. Geng, J. Wang, and W. Xu, "Preparation and performance of nano HMX/TNT cocrystals," Propellants, Explosives, Pyrotechnics, vol. 40, no. 5, pp. 652-658, 2015.

[7] Y. Liu, C. An, J. Luo, and J. Wang, "High-density HNIW/TNT cocrystal synthesized using a green chemical method," Acta Crystallographica Section B: Structural Science, vol. 74, no. 4, pp. 385-393, 2018.

[8] J.-W. Jung and K.-J. Kim, "Effect of supersaturation on the morphology of coated surface in coating by solution crystallization," Industrial and Engineering Chemistry Research, vol. 50, no. 6, pp. 3475-3482, 2011.

[9] J. Liu, X. Bao, Y. Rong et al., "Preparation of nano-RDX-based PBX and its thermal decomposition properties," Journal of Thermal Analysis and Calorimetry, vol. 131, no. 3, pp. 26932698, 2018.

[10] Z. Ma, B. Gao, P. Wu et al., "Facile, continuous and large-scale production of core-shell HMX@TATB composites with superior mechanical properties by a spray-drying process," RSC Advances, vol. 5, no. 27, pp. 21042-21049, 2015.

[11] N. Liu, B. Duan, X. Lu et al., "Preparation of CL-20/DNDAP cocrystals by a rapid and continuous spray drying method: an alternative to cocrystal formation," CrystEngComm, vol. 20 , no. 14, 2018.

[12] D. Wang, B. Gao, G. Yang, F. Nie, and H. Huang, "Preparation of Cl-20 explosive nanoparticles and their thermal decomposition property," Journal of Nanomaterials, vol. 2016, 7 pages, 2016.

[13] G. Zeng, W. Pang, and J. Zhou, "Preparation and characterization of TATB based nanocomposites," Procedia Engineering, vol. 102, pp. 610-614, 2015.

[14] P. Li, K. Liu, D. Ao et al., "A low-sensitivity nanocomposite of CL-20 and TATB," Crystal Research and Technology, vol. 53, no. 11, 2018.

[15] W. Xu, Z. Pang, J. Wang, C. Ping, J. Wang, and J. Peng, "Preparation and characterization of TATB/VitonA nanocomposites," Journal of Nanomaterials, vol. 2018, 9 pages, 2018.

[16] H. Xu, X. Duan, H. Li, and C. Pei, "A novel high-energetic and good-sensitive cocrystal composed of CL-20 and TATB by a rapid solvent/non-solvent method," RSC Advances, vol. 5, no. 116, pp. 95764-95770, 2015.

[17] W. Ji, X. Li, J. Wang, B. Ye, and C. Wang, "Preparation and characterization of the solid spherical HMX/F2602by the suspension spray-drying method," Journal of Energetic Materials, vol. 34, no. 4, pp. 357-367, 2016.

[18] C. An, H. Li, X. Geng, J. Li, and J. Wang, "Preparation and properties of 2,6-diamino-3,5-dinitropyrazine-1-oxide based nanocomposites," Propellants, Explosives, Pyrotechnics, vol. 38, no. 2, pp. 172-175, 2013.

[19] J. Wang, B. Ye, C. An, B. Wu, H. Li, and Y. Wei, "Preparation and properties of surface-coated HMX with Viton and 
graphene oxide," Journal of Energetic Materials, vol. 34, no. 3, pp. 235-245, 2016.

[20] A. Taguet, B. Ameduri, and B. Boutevin, "Crosslinking of vinylidene fluoride-containing Fluoropolymers," Advances in Polymer Science, vol. 184, pp. 127-211, 2005.

[21] C. Roth and J. Gebhart, "Aqueous droplet sizing by inertial classification," Particle and Particle Systems Characterization, vol. 13, no. 3, pp. 192-195, 1996.

[22] L. Liao, Y. B. Li, J. Xu et al., "Competitive influence of hydrogen bonding and van der Waals interactions on selfassembled monolayers of stilbene-based carboxylic acid derivatives," The Journal of Physical Chemistry C, vol. 118, no. 49, pp. 28625-28630, 2014.

[23] Q. L. Yan, S. Zeman, and A. Elbeih, "Thermal behavior and decomposition kinetics of Viton A bonded explosives containing attractive cyclic nitramines," Thermochimica Acta, vol. 562, pp. 56-64, 2013.

[24] Z. Yang, J. Li, B. Huang, S. Liu, Z. Huang, and F. Nie, "Preparation and properties study of core-shell CL-20/TATB composites," Propellants Explosives Pyrotechnics, vol. 39, no. 1, pp. 51-58, 2014.

[25] S. P. Felix, G. Singh, A. K. Sikder, and J. P. Aggrawal, "Studies on energetic compounds: part 33: thermolysis of keto-RDX and its plastic bonded explosives containing thermally stable polymers," Thermochimica Acta, vol. 426, no. 1-2, pp. 53-60, 2005. 